Published in final edited form as:

Clin Cancer Res. 2012 August 1; 18(15): 4026-4036. doi:10.1158/1078-0432.CCR-12-0343.

\title{
Molecular Profiling Reveals Low- and High-Grade Forms of Primary Melanoma
}

\author{
Katja Harbst ${ }^{1,2}$, Johan Staaf ${ }^{1,2}$, Martin Lauss ${ }^{1,2}$, Anna Karlsson ${ }^{1}$, Anna Måsbäck ${ }^{3}$, Iva \\ Johansson $^{3}$, Pär-Ola Bendahl ${ }^{1}$, Johan Vallon-Christersson ${ }^{1,2}$, Therese Törngren ${ }^{1}$, Henrik \\ EkedahI $^{1}$, Jürgen Geisler ${ }^{5}$, Markus Höglund ${ }^{1,2}$, Mattias Ringnér ${ }^{1,2}$, Lotta Lundgren ${ }^{1}$, Karin \\ Jirström $^{3}$, Håkan Olsson ${ }^{1}$, Christian Ingvar ${ }^{4}$, Åke Borg ${ }^{1,2}$, Hensin Tsao ${ }^{6,7}$, and Göran \\ Jönsson ${ }^{1,2}$ \\ ${ }^{1}$ Department of Oncology, Clinical Sciences, Lund University, Lund, Sweden \\ ${ }^{2}$ CREATE Health Strategic Center for Clinical Cancer Research, Lund University, Lund, Sweden \\ ${ }^{3}$ Department of Pathology, Lund University, Lund, Sweden \\ ${ }^{4}$ Department of Surgery, Lund University, Lund, Sweden \\ ${ }^{5}$ Department of Oncology, Akershus University Hospital, University of Oslo, Norway \\ ${ }^{6}$ Department of Dermatology, Harvard Medical School, Massachusetts General Hospital, Boston, \\ Massachusetts \\ ${ }^{7}$ Wellman Center for Photomedicine, MGH Cancer Center, Massachusetts General Hospital, \\ Boston, Massachusetts
}

\section{Abstract}

Purpose-For primary melanomas, tumor thickness, mitotic rate, and ulceration are well-laid cornerstones of prognostication. However, a molecular exposition of melanoma aggressiveness is critically missing. We recently uncovered a four-class structure in metastatic melanoma, which predicts outcome and informs biology. This raises the possibility that a molecular structure exists

\footnotetext{
(C) 2012 American Association for Cancer Research.

Corresponding Authors: Göran Jönsson, Department of Oncology, Clinical Sciences, Lund University, Lund 22185, Sweden. Phone: 46-46-222-1444; Fax: 46-46-147327; goran_b.jonsson@med.lu.se; and Hensin Tsao, Wellman Center for Photomedicine/Department of Dermatology, Harvard Medical School, Boston, MA. Phone: 617-726-9569; Fax: 617-726-1206; htsao@ partners.org.

Note: Supplementary data for this article are available at Clinical Cancer Research Online (http://clincancerres.aacrjournals.org/).

Disclosure of Potential Conflicts of Interest

No potential conflicts of interest were disclosed.

Authors' Contributions

Conception and design: K. Harbst, J. Staaf, J. Vallon-Christersson, J. Geisler, L. Lundgren, C. Ingvar, A. Borg, H. Tsao, G. Jönsson Development of methodology: A. Karlsson, J. Vallon-Christersson, H. Tsao

Acquisition of data (provided animals, acquired and managed patients, provided facilities, etc.): K. Harbst, H. Ekedahl, K. Jirström, H. Olsson, C. Ingvar

Analysis and interpretation of data (e.g., statistical analysis, biostatistics, computational analysis): K. Harbst, J. Staaf, M. Lauss, I. Johansson, P.-O. Bendahl, J. Vallon-Christersson, T. Törngren, J. Geisler, M. Höglund, M. Ringnér, L. Lundgren, C. Ingvar, H.

Tsao, G. Jönsson

Writing, review, and/or revision of the manuscript: K. Harbst, J. Staaf, P.-O. Bendahl, J. Vallon-Christersson, J. Geisler, M. Höglund, M. Ringnér, L. Lundgren, K. Jirström, H. Olsson, C. Ingvar, A. Borg, H. Tsao, G. Jönsson

Administrative, technical, or material support (i.e., reporting or organizing data, constructing databases): K. Harbst, A.

Karlsson, J. Vallon-Christersson, C. Ingvar, G. Jönsson

Study supervision: C. Ingvar, H. Tsao, G. Jönsson

Histologic interpretation and selection of tumor material and discussions on the study design: A. Måsbäck
} 
even in the early stages of melanoma and that molecular determinants could underlie histophenotype and eventual patient outcome.

Experimental Design-We subjected 223 archival primary melanomas to a horizontally integrated analysis of RNA expression, oncogenic mutations at 238 lesions, histomorphometry, and survival data.

Results-Our previously described four-class structure that was elucidated in metastatic lesions was evident within the expression space of primary melanomas. Because these subclasses converged into two larger prognostic and phenotypic groups, we used the metastatic lesions to develop a binary subtype-based signature capable of distinguishing between "high" and "low" grade forms of the disease. The two-grade signature was subsequently applied to the primary melanomas. Compared with low-grade tumors, high-grade primary melanomas were significantly associated with increased tumor thickness, mitotic rate, ulceration (all $P<0.01$ ), and poorer relapse-free ( $\mathrm{HR}=4.94 ; 95 \% \mathrm{CI}, 2.84-8.59)$, and overall ( $\mathrm{HR}=3.66$; 95\% CI, 2.40-5.58) survival. High-grade melanomas exhibited elevated levels of proliferation and BRCA1/DNA damage signaling genes, whereas low-grade lesions harbored higher expression of immune genes. Importantly, the molecular-grade signature was validated in two external gene expression data sets.

Conclusions-We provide evidence for a molecular organization within melanomas, which is preserved across all stages of disease.

\section{Introduction}

One fundamental observation in melanoma that has withstood decades of epidemiologic scrutiny is the inverse relationship between tumor thickness and prognosis (1). Despite widespread replication and clinical adoption, the etiologic basis for this correlation is largely unknown. Although time-to-diagnosis represents one possible explanation, recent analyses challenge the notion that primary melanomas strictly abide by volumetric rules and that lesions progress monotonically toward fatality because of detection failure. Alternative views include the possibility that lethal melanomas may reflect a class of aggressive tumors that are unrecognizable by microscopic means. For instance, the mitotic rate-an index of proliferative activity - is significantly higher in thick compared to thin melanomas and directly correlated with the presence of ulceration-a negative predictor of outcome $(1,2)$. Taken together, these long-held observations suggest that there may in fact be a "highgrade" form of melanoma that is thick, mitogenic, and ulcerated and that reflects a context of aggression rather than time in corpus. The biologic basis of such aggressive (i.e., lethal) melanomas represents a major conceptual gap that remains unfilled.

Early efforts to resolve melanoma into molecular profiles were fraught with challenges (reviewed in refs. 3, 4). First generation platforms required robust RNA, which was only derivable from frozen tissue and thus inaccessible to formalin-fixed archival specimens. Because primary melanomas are largely fixed and paraffinized for diagnosis, the vast majority of studies used metastatic melanoma tissue. Thus, early molecular subtyping was not fully validated on primary melanomas. Winnepenninckx and colleagues (5) successfully conducted unsupervised hierarchical clustering and recovered a 2-class structure that was marginally associated with prognosis. Although the study was informative, it was limited by the availability of frozen specimens and the inclusion of stages III and IV patients, which made it impossible to test for preservation of the molecular classifier across different stages of disease. With advent of the cDNA-mediated annealing, selection, extension, and ligation (DASL) technique, formalin-fixed, paraffin embedded (FFPE) archival tissue became viable substrates for analysis. Several recent studies using DASL and a fixed cancer panel microarray led to the identification of osteopontin, RAD51, RAD52, and TOP2A as 
prognostic biomarkers in primary melanomas $(6,7)$. What is critically needed in primary melanoma is a horizontally integrated analysis, which incorporates molecular and genetic exposition with known pathologic indicators and annotated outcomes. Furthermore, if molecular forms of melanoma exist and are innate to the tumor, the distinct signatures should be recoverable from various stages of melanoma.

To explore unrecognized structures within the melanoma expression space, we recently conducted an unsupervised analysis of stage IV melanoma and delineated 4 distinct molecular subtypes. Superimposition of these signatures upon available outcomes data showed that an "immune response" gene signature was associated with a significantly better prognosis than a "proliferative" gene signature (8).

Furthermore, the 4-class organization was also validated in an independent set of stage III melanoma samples $(8,9)$. Here, we applied whole genome-DASL to a fully annotated set of 223 FFPE primary melanomas and provide evidence for high- and low-grade forms of the disease that are derived from the 4-class structure and that are marked by differences in molecular profile and level of aggressiveness.

\section{Patients and Methods}

\section{Clinical samples and RNA extraction from FFPE tissue blocks}

This study was approved by the local ethics committee of the Lund University, Lund, Sweden (Dnr 191/2007). FFPE tissue blocks from primary cutaneous melanomas (CM, \0.8 $\mathrm{mm}$ ) diagnosed from 1995 to 2002 were collected from the Department of Pathology, Skåne University Hospital, Sweden $(n=205)$ along with available acral lentiginous melanomas (ALM) and mucosal melanomas $(n=18)$ from the archives at the Department of Pathology (Table 1). Conventional clinical and clinic-pathologic parameters were retrieved from clinical chart records. Hematoxylin-eosin sections were confirmed to contain at least $70 \%$ tumor cells by a pathologist (A. Måsbäck). On average, three 10-mm sections were cut from each block and subjected to macro dissection. The RNA was extracted using High Pure RNA Paraffin Kit (Roche Diagnostics) and genomic DNA using the QIAamp DNA FFPE kit (Qiagen). Tumor-infiltrating lymphocytes (TIL) were determined according to Clark and colleagues (10) and mitoses were calculated according to the American Joint Committee on Cancer (AJCC) staging system (1).

\section{Gene expression analysis of melanoma}

Four molecular subtypes in stage IV melanomas were recently identified (8). In this study, we refined the subtype-specific centroids corresponding to the pigmentation, proliferative, normal-like, and high immune response groups in the stage IV melanomas. Primary melanomas were analyzed using the WG-DASL assay (Illumina; herein referred to as theWG-DASL set). Further details on data processing and the 4-class and 2-grade SAM analyses are provided in Supplementary Methods. A flowchart describing the analysis is provided in Supplementary Fig. S1.

\section{Mutation screening using the Sequenom OncoCarta Panel v1}

We used the OncoCarta Panel v1 (Sequenom) to determine the mutation frequency of 19 oncogenes (238 mutations), including $B R A F$ and $N R A S$ in 129 melanomas. Briefly, we used 500 ng FFPE DNA for the complete analysis of 24 primer mixes and 32 samples were analyzed for 12 primer mixes for each 384-well plate. Genomic DNA (100 ng) from FFPE tissue was amplified using BioScore (Enzo Life Sciences) and $500 \mathrm{ng}$ amplified DNA was subsequently used in the Sequenom analysis. Comparison between amplified and unamplified FFPE DNA were checked and revealed identical results. Results were analyzed 
using the Type v1.4 software using an allele mutation frequency of $10 \%$ as cutoff. Manual inspection of suggested mutations was conducted.

\section{Outcome analysis and prognostic model in melanoma}

Univariate and multivariate Cox survival analyses were conducted using overall survival (OS) or relapse-free survival (RFS) as endpoints. Proportional hazards assumptions were checked graphically. Kaplan-Meier curves were compared with the log-rank test using fulltime follow-up. The prognostic value of molecular grading was compared with subgroups stratified by age ( $\leq$ or $>50$ years), Breslow thickness ( $\leq$ or $>2 \mathrm{~mm}$ ), gender, mitotic rate ( $\leq$ or $>6$ mitoses $/ \mathrm{mm}^{2}$ ), TIL (absent or present), and type of melanoma [nodular melanoma (NM) or superficial spreading melanoma (SSM)]. For the subgroup analysis of melanomas $\geq 2 \mathrm{~mm}$, thickness was stratified $\leq$ or $>4 \mathrm{~mm}$. Multivariate analysis was adjusted for AJCC stage (stages I and II) and molecular grade (high- and low-grade). All statistical analyses were conducted using R software.

\section{Pathway analysis}

Gene ontology analysis was conducted using the web-based software DAVID (11). Standard default settings were used in the ingenuity pathway analysis (IPA) software. Gene expression centroids used to predict high- and low-grade melanomas were used as input gene list.

\section{Array CGH and genomic imbalance calculation}

Array CGH and calculation of genomic imbalances were carried out as described earlier (8).

\section{Results}

\section{Molecular subtypes in primary melanoma}

We previously conducted unsupervised global hierarchical clustering of stage IV melanomas and uncovered a hidden 4-class molecular structure that was also evident in stage III melanomas upon independent validation (8). We thus set out to determine if these molecular signatures are retained in primary CMs by WG-DASL analysis of a cohort of 223 cases (Table 1 ). Of the 223 primary melanomas ( $N=210$ cutaneous, $N=13$ mucosal) analyzed in the WG-DASL set, $26 \%$ were high-immune response, $28 \%$ were normal-like, $37 \%$ were pigmentation, $7 \%$ were proliferative; only $2 \%$ were unclassified (Fig. 1A and Table 1). These findings indicate that the 4 molecular classes are preserved across all stages of melanoma.

Also consonant with our previous analysis of stages III and IV tumors, the high-immune and normal-like subtypes were associated with significantly better OS $\left(P=2.32 \times 10^{-8}\right.$, log-rank test $)$ and RFS $\left(P=1.98 \times 10^{-7}, \log\right.$-rank test $)$ than the pigmentation and proliferative subtypes (Fig. 1B and C). However, closer examination of the distribution of histologic parameters (Table 1) and the similarities in survival (Fig. 1A and B) points at a potential convergence of the 4-class structure into 2 distinct forms of the disease-a "high-grade" class of aggressive tumors (proliferative/pigmentation) and a "low-grade" class of more indolent lesions (high-immune/normal-like). This is further supported by investigation of specific gene expression patterns. Specifically, cell-cycle associated genes were upregulated in both pigmentation and proliferative subtypes, whereas in the normal-like and highimmune response subtypes immune-response associated genes were upregulated (Fig. 1A). This raised the possibility of creating a 2-class "molecular grading" system, which could be both biologically informative and clinically predictive of outcome. Another reason for constructing a 2-class signature is because of statistical power, which is increased when dividing the cohort in low- and high-grade melanomas instead of the 4-group structure. 


\section{High- and low-grade forms of primary melanoma}

To construct the binary classifier (i.e., "high-grade" and "low-grade"), we used the original high-immune/normal-like classes and the pigmentation/proliferative classes from the 57 stage IV melanomas (GSE22155). SAM analysis identified 1,864 genes distinguishing the combined high-immune/normal-like group from the pigmentation/proliferative group in the stage IV melanoma set. When this 2-group signature was applied to the 223 primary melanomas using nearest centroid classification, we found that 103 cases belonged to "lowgrade" category, 93 cases to "high-grade" group, and 27 cases belonged to neither group (Fig. 1D and Table 1). Thus, in subsequent analyses, we focused on the 196 classifiable cases.

The median follow-up duration for our cohort was 10 years (range $0.1-14.5$ years). As shown in Fig. 1E and F, there were highly significant differences between the low- and highgrade melanomas both in $\mathrm{OS}\left(\mathrm{HR}=3.66 ; 95 \% \mathrm{CI}, 2.40-5.58 ; P=2.48 \times 10^{-11}\right)$ and in RFS (HR $=4.94 ; 95 \%$ CI, $\left.2.84-8.59 ; P=9.16 \times 10^{-10}\right)$. As outlined in Table 1, high-grade tumors were significantly associated with thicker tumors, increased AJCC stage, higher mitotic rates ( $\ 6$ mitoses $/ \mathrm{mm}^{2}$ ), a lower prevalence of tumors with brisk TILs, and a greater frequency of nodular melanomas. In other words, primary melanomas with the high-grade signature possess many of the known phenotypic indicators of poor outcome. Having applied the 2-grade signature to the WG-DASL set, we also externally validated the 2-grade signature on 2 independent public data sets. Reassuringly, high-grade tumors were associated with poorer outcomes (Fig. 2$)$ in both stage III melanomas $(N=29 ; P=0.003$, log-rank test; ref. 9) as well as primary melanomas ( $N=79 ; P=0.04$, log-rank test; ref. 5). In the primary melanoma data set (5), we also observed a significant correlation between ulceration and thickness and the molecular grade $(P=0.04$ and 0.02 , Fisher's exact test and Mann-Whitney test, respectively). Taken together, high- and low-grade forms of melanoma seem to be recoverable from all stages of disease, to be consistently predictive of survival, and to be reproducible across technical platforms and independent data sets.

We next determined if the high- and low-grade forms were recognizable within clinical subgroups of melanoma in the WG-DASL set. Among the various features examined (CM, subtype, and gender; Fig. 3), high-grade melanomas consistently exhibited worsened RFS and OS compared with their low-grade counterparts. For the most established prognostic indicators, high-grade tumors appeared to be more mitotically active, thicker, and more ulcerated than low-grade tumors (Fig. 4A). Because of the apparent relationship between tumor grade and thickness, we next assessed only melanomas $\geq 2 \mathrm{~mm}$ and found that both molecular forms were still preserved and that a high-grade signature still predicted worse RFS (HR = 2.59; 95\% CI, 1.09-6.16; Fig. 4C) and OS (HR = 3.10; 95\% CI, 1.40-6.85; Fig. 4D). We proceeded by investigating this observation in the Winnepenninckx data set that includes 83 primary melanomas (5). Importantly, we could confirm the prognostic impact of molecular grade in thick melanomas ( $\mathrm{HR}=2.56$; 95\% CI, 1.08-6.07; Fig. 4E). Notably, tumor thickness, mitoses, TILs, and ulceration were not predictive of prognosis in melanomas $\geq 2 \mathrm{~mm}$ in the Winnepenninckx data set $(P>0.05$, log-rank test). Thus, tumor thickness is unlikely to account for the entire discriminatory capabilities of molecular grading.

Having established the prognostic power of the grade signature and its association with conventional clinical parameters, we next set out to compare the grade signature with other prognostic factors (Supplementary Table $\mathrm{S} 1$ ). For the entire cohort, univariate analysis (RFS) revealed that molecular grading ( $\mathrm{HR}=4.94 ; 95 \% \mathrm{CI}, 2.84-8.59)$ was one of the most significant determinants of outcome along with tumor thickness (HR $=5.30$; 95\% CI, 2.999.41), mitotic rate ( $\mathrm{HR}=2.93$; 95\% CI, 1.65-5.09), and TILs ( HR $=0.56$; 95\% CI, 0.34 $0.92)$. For melanomas $\geq 2 \mathrm{~mm}$, only molecular grade retained its discriminatory power ( $\mathrm{HR}=$ 
3.10; 95\% CI, 1.4-6.85). The same kind of analysis was not possible in tumors $<2 \mathrm{~mm}$ because of the low number of events in this group.

Finally, we adjusted for molecular grading and staging (stage I vs. stage II) in a Cox multivariate model and found that the 2-grade signature adds independent prognostic value in the whole cohort $(\mathrm{HR}=2.51 ; 95 \% \mathrm{CI}, 1.11-5.67)$, as did the AJCC staging system ( $\mathrm{HR}=$ 2.96; 95\% CI, 1.12-7.38). Taken together, these results indicate that molecular tumor grade is an independent prognostic factor in primary melanoma.

\section{Mutation screening of low- and high-grade melanomas}

The presence of recurrent oncogenic mutations in melanoma may further influence the molecular structure clarified from RNA profiling. Consequently, we analyzed 238 somatic mutations ( 19 oncogenes) in 129 of the 223 primary melanomas $(N=52$, high-grade tumors; $N=62$, low-grade tumors). In this cohort, 38 cases (30\%) harbored $B R A F$ mutations (29 were V600E, 6 were V600K, 1 was V600R, 1 was K601E, and 1 case was L597S), 23 tumors (18\%) had NRAS mutations (all Q61 changes) and 6 specimens (5\%) contained MET mutations; there were also single cases of CDK4, KIT, and EGFR mutations.

We found a higher frequency of $B R A F$ mutations among low-grade compared with highgrade melanomas (39\% vs. $19 \% ; P=0.03$, Fisher's exact test; Table 1 ) and an inverse relationship, although not statistically significant, for $N R A S$ mutations $(11 \%$ vs. $25 \% ; P=$ 0.06 , Fisher's exact test). $B R A F$ mutations were more common in melanomas $<2 \mathrm{~mm}$ compared with those $\geq 2 \mathrm{~mm}$ ( $38 \%$ vs. $23 \%, P=0.06$, Fisher's exact test) - a finding that is consistent with a previous report (12). After adjusting for Breslow thickness in a logistic regression model, no significant difference in $B R A F$ or $N R A S$ mutation frequency was observed between low- and high-grade melanomas ( $P=0.18$ and 0.16 , respectively). However, even among $B R A F$-mutated melanomas, the grade signature is still predictive of outcome (Supplementary Fig. S2; $P=0.05, \log$-rank test). Although the number of cases was small, the overall picture suggests that low-grade tumors tend to harbor $B R A F$ mutations and that the high-grade tumors were associated with both $N R A S$ and $B R A F$ mutations. These findings do indicate, however, that the molecular grades are unlikely to be driven by $B R A F$ and NRAS mutations alone.

\section{Defining biologic characteristics of the predictive gene signature}

To obtain a more comprehensive view of the biologic systems underlying the molecular grade signature, we conducted gene ontology analysis of the predictive genes. This approach highlighted wound response, immune response, DNA repair, and cell cycle as biologic processes (Fig. 1D). It is worth noting that the observed expression of immune signature genes is supported by an association between a brisk pattern of TILs and the low-grade form of melanoma (Fig. 5 and Table 1).

Beyond ontology, IPA identified DNA damage/repair and cell-cycle processes as important pathways (Supplementary Table S2). Notably, "Hereditary Breast Cancer signaling" and "BRCA1 DNA Damage Response" emerged as the 2 most significant networks $\left(P=10^{-10}\right.$ and $10^{-8}$, respectively) associated with high-grade tumors. In contrast, there were no strong pathway links to low-grade melanomas, although upregulation of the NOTCH pathway was detected by IPA.

There are several potential biologic rationales for the relationship between high-grade melanomas and the BRCA1/DNA damage response. One possibility is that high-grade melanomas harbor more genomic derangements thereby stimulating an ongoing damage response. To test this hypothesis, we analyzed available array CGH data from the GSE22155 data collection and indeed found a significantly increased level of genomic imbalance 
among high-grade metastatic melanomas (Fig. 5A; $P<0.01$, Mann-Whitney test). Although we had array CGH data only on a small fraction of the primary cases $(n=12)$, a trend toward greater genomic instability among high-grade primary melanomas was observed $(P=$ 0.16 , Mann-Whitney test). Because an acral melanoma [a subtype known to be particularly affected by chromosomal imbalances (13)] was classified as low-grade and was the single outlier among the low-grade melanomas, we conducted a sensitivity analysis and subsequently found a significant difference in genomic instability $(P=0.02$, Mann-Whitney test). However, these data should be interpreted with caution, because the number of tumors with complementary array CGH data was small.

Another possibility for the connection between DNA damage response and high-grade melanomas reflects coordinated transcription. When the 196 samples were ranked by signature correlation from low to high grade, there was an increasing gradient of expression for MITF, its upstream regulator SOX10 and its downstream targets, TYR and MLANA (Fig. 5B). Expression levels of BRCA1 DNA damage response pathway genes, such as $B R C A 1, A T M$, and FANCA, showed a similar trend with MITF and its targets. This finding is particularly intriguing given the recent report that BRCA1, FANCA, and ATM are transcriptional targets of MITF $(14,15)$. As expected, immune-related genes, such as $C D 3 E$, $C D 3 D$, and $L C K$, followed an inverse pattern of expression.

\section{Discussion}

Through molecular profiling, we provide evidence of low- and high-grade forms of primary melanoma. Compared with low-grade melanomas, high-grade lesions are thicker, more mitotically active and more ulcerative - all of which are known features of worsened survival. There are several notable elements in this study. First, both the 4-class structure and the molecular grading have now been recovered from melanomas in all stages of progression, and the 2-grade signature was independently validated in 2 gene expression data sets. Thus, this and previous study (8) point to a molecular architecture that is recognizable even at the earliest stages of melanomagenesis. Second, with 18,000 genes in our molecular palette, this is the broadest application of the WG-DASL technology for archival melanoma specimens to date. In addition, we cross-examined 238 mutations in 19 oncogenes to create a strong horizontally-integrated data platform. With continued optimization of more refined techniques, barriers to the molecular analysis of primary melanomas should be viewed as surmountable. Third, with more than 200 primary melanomas analyzed, the study is of sufficient size to show the power of molecular grading in predicting outcome even after adjusting for AJCC staging. Furthermore, molecular grading has immediate clinical implications. In general, most thin melanomas eventuate into a benign course. However, the outcomes of melanomas $\geq 2 \mathrm{~mm}$ remain less certain (1). The decision to administer potentially toxic and costly adjuvant therapies in patients with thick melanomas could be made more judiciously with these molecular signatures. In this study, we show that the molecular grade signature is superior to tumor thickness in predicting prognosis among thick melanomas ( $\geq 2 \mathrm{~mm}$ ). Although additional replication and refinement are required, one obvious possibility is to focus treatments on patients with high-grade melanomas.

Beyond the possible clinical applications of tumor grading, the molecular underpinnings of the subclassification are also provocative. The largest subgroup in primary melanomas is grounded in an MITF-based signature, suggesting that this subgroup is highlighted by a crucial biologic driver. It is interesting to note, for instance, that MITF and AKT3 are overexpressed in the high-grade melanomas, which are associated with proliferative tumors. Both genes have also been shown to be amplified or upregulated in melanoma $(16,17)$ and may play significant roles in melanoma mitogenicity along with other species revealed by 
these signatures. Even more exciting is the nexus between tumor grade, MITF, and DNA damage response. In a smaller earlier series, Kaufmann and colleagues adopted a supervised approach and also identified DNA repair genes, including BRCA1, in metastasis-prone melanomas (18). Jewell and colleagues found that overexpression of DNA repair genes $R A D 51$ and $R A D 52$ were also associated with an increased risk of relapse (7). More recently, a report identified $B R C A 1$ and $A T M$ - two components of the upregulated pathway in high-grade tumors - as targets of MITF (14). Furthermore, in ChIP-Seq experiments, BRCA1 and FANCA promoters (high-grade genes; Fig. 5B) were shown to be occupied and regulated by MITF (15). Through this analysis and others recently published, a novel intersection is beginning to emerge between MITF and DNA repair, particularly the BRCA1 pathway, although the mechanisms are far from understood.

The physiologic determinants of low-grade melanomas are less well formulated than the high-grade ones. The positive survival impact of the immune-gene signature, which is a critical component of low-grade melanomas, was shown in earlier analyses $(8,9)$ and reproduced in this study. In primary melanomas, the presence of TILs is an established favorable prognostic factor $(19,20)$ and is also closely associated with our high-immune subclass.

A higher frequency of $N R A S$ mutations was found in high-grade melanomas though this was far from absolute. $B R A F$ mutations were not related to RFS in a recent study (21), although there have been reports of specific histomorphometric features associated with $B R A F$ mutations (22). It is possible that $B R A F$ and $N R A S$ define the nature of the proliferative apparatus but do not orchestrate the metastatic program that determines tumor grade and melanoma aggressiveness.

There are some limitations that are practically tied to this study. The case samples for this study were derived from a single institution in Sweden. Although it is imperative that the findings be replicated in other populations, one could also argue that there is greater consistency in terms of diagnosis and treatment and more reproducibility if a homogeneous population is analyzed. It is reassuring however, that our molecular grades were validated in other publicly available independent data sets. Also, despite a reasonable sample size, it has not been possible to correlate melanoma grade with all known pathologic features. Efforts are underway to enlarge the cohort size, to involve additional case sites and to further explore phenotype-genotype correlates.

In conclusion, we undertook a molecular approach to melanoma and uncovered a hidden structure that is technically reproducible, biologically based and clinically relevant. The formulation of high- and low-grade melanomas represents a synthesis of various technological capabilities currently available in cancer profiling. Further replication in other populations will ideally lead to a more generalized molecular tool, which can be deployed to assist clinicians in both prognostic and therapeutic decision making.

\section{Supplementary Material}

Refer to Web version on PubMed Central for supplementary material.

\section{Acknowledgments}

\section{Grant Support}

This work was supported by the Swedish Cancer Society, the Crafoord Foundation the Mrs. Berta Kamprad Foundation, the Gunnar Nilsson Cancer Foundation, the Lund University Hospital Research Funds, Lund University Medical Faculty/ALF, BioCare, and the Gustav Vth Jubilee Foundation. This activity was also 
supported in part by the U.S. NIH (K24 CA149202 and P50 CA93683) and by the generous donors to the MGH Millennium Melanoma Fund.

\section{References}

1. Balch CM, Gershenwald JE, Soong SJ, Thompson JF, Atkins MB, Byrd DR, et al. Final version of 2009 AJCC melanoma staging and classification. J Clin Oncol. 2009; 27:6199-6206. [PubMed: 19917835]

2. Thompson JF, Soong SJ, Balch CM, Gershenwald JE, Ding S, Coit DG, et al. Prognostic significance of mitotic rate in localized primary cutaneous melanoma: an analysis of patients in the multi-institutional American Joint Committee on Cancer melanoma staging database. J Clin Oncol. 2011; 29:2199-2205. [PubMed: 21519009]

3. Hoek KS. DNA microarray analyses of melanoma gene expression: a decade in the mines. Pigment Cell Res. 2007; 20:466-484. [PubMed: 17935490]

4. Nambiar S, Mirmohammadsadegh A, Hengge UR. Cutaneous melanoma: fishing with chips. Curr Mol Med. 2008; 8:235-243. [PubMed: 18473822]

5. Winnepenninckx V, Lazar V, Michiels S, Dessen P, Stas M, Alonso SR, et al. Gene expression profiling of primary cutaneous melanoma and clinical outcome. J Natl Cancer Inst. 2006; 98:472482. [PubMed: 16595783]

6. Conway C, Mitra A, Jewell R, Randerson-Moor J, Lobo S, Nsengimana J, et al. Gene expression profiling of paraffin-embedded primary melanoma using the DASL assay identifies increased osteopontin expression as predictive of reduced relapse-free survival. Clin Cancer Res. 2009; 15:6939-6946. [PubMed: 19887478]

7. Jewell R, Conway C, Mitra A, Randerson-Moor J, Lobo S, Nsengimana J, et al. Patterns of expression of DNA repair genes and relapse from melanoma. Clin Cancer Res. 2010; 16:52115221. [PubMed: 20705614]

8. Jonsson G, Busch C, Knappskog S, Geisler J, Miletic H, Ringner M, et al. Gene expression profiling-based identification of molecular subtypes in stage IV melanomas with different clinical outcome. Clin Cancer Res. 2010; 16:3356-3367. [PubMed: 20460471]

9. Bogunovic D, O'Neill DW, Belitskaya-Levy I, Vacic V, Yu YL, Adams S, et al. Immune profile and mitotic index of metastatic melanoma lesions enhance clinical staging in predicting patient survival. Proc Natl Acad Sci U S A. 2009; 106:20429-20434. [PubMed: 19915147]

10. Clark WH Jr, Elder DE, Guerry D IV, Braitman LE, Trock BJ, Schultz D, et al. Model predicting survival in stage I melanoma based on tumor progression. J Natl Cancer Inst. 1989; 81:1893-1904. [PubMed: 2593166]

11. Huang da W, Sherman BT, Lempicki RA. Bioinformatics enrichment tools: paths toward the comprehensive functional analysis of large gene lists. Nucleic Acids Res. 2009; 37:1-13. [PubMed: 19033363]

12. Devitt B, Liu W, Salemi R, Wolfe R, Kelly J, Tzen CY, et al. Clinical outcome and pathological features associated with NRAS mutation in cutaneous melanoma. Pigment Cell Melanoma Res. 2011; 24:666-672. [PubMed: 21615881]

13. Curtin JA, Fridlyand J, Kageshita T, Patel HN, Busam KJ, Kutzner H, et al. Distinct sets of genetic alterations in melanoma. N Engl J Med. 2005; 353:2135-2147. [PubMed: 16291983]

14. Beuret L, Ohanna M, Strub T, Allegra M, Davidson I, Bertolotto C, et al. BRCA1 is a new MITF target gene. Pigment Cell Melanoma Res. 2011; 24:725-727. [PubMed: 21501420]

15. Strub T, Giuliano S, Ye T, Bonet C, Keime C, Kobi D, et al. Essential role of microphthalmia transcription factor for DNA replication, mitosis and genomic stability in melanoma. Oncogene. 2011; 30:2319-2332. [PubMed: 21258399]

16. Stahl JM, Sharma A, Cheung M, Zimmerman M, Cheng JQ, Bosenberg MW, et al. Deregulated Akt3 activity promotes development of malignant melanoma. Cancer Res. 2004; 64:7002-7010. [PubMed: 15466193]

17. Garraway LA, Widlund HR, Rubin MA, Getz G, Berger AJ, Ramaswamy S, et al. Integrative genomic analyses identify MITF as a lineage survival oncogene amplified in malignant melanoma. Nature. 2005; 436:117-122. [PubMed: 16001072] 
18. Kauffmann A, Rosselli F, Lazar V, Winnepenninckx V, Mansuet-Lupo A, Dessen P, et al. High expression of DNA repair pathways is associated with metastasis in melanoma patients. Oncogene. 2008; 27:565-573. [PubMed: 17891185]

19. Burton AL, Roach BA, Mays MP, Chen AF, Ginter BA, Vierling AM, et al. Prognostic significance of tumor infiltrating lymphocytes in melanoma. Am Surg. 2011; 77:188-192. [PubMed: 21337878]

20. Taylor RC, Patel A, Panageas KS, Busam KJ, Brady MS. Tumor-infiltrating lymphocytes predict sentinel lymph node positivity in patients with cutaneous melanoma. J Clin Oncol. 2007; 25:869875. [PubMed: 17327608]

21. Long GV, Menzies AM, Nagrial AM, Haydu LE, Hamilton AL, Mann GJ, et al. Prognostic and clinicopathologic associations of oncogenic BRAF in metastatic melanoma. J Clin Oncol. 2011; 29:1239-1246. [PubMed: 21343559]

22. Broekaert SM, Roy R, Okamoto I, van den Oord J, Bauer J, Garbe C, et al. Genetic and morphologic features for melanoma classification. Pigment Cell Melanoma Res. 2010; 23:763770. [PubMed: 20874733] 


\section{Translational Relevance}

Over the years, clinical markers of melanoma behavior have been slowly unveiled through rounds of phenotypic analyses. However, the molecular composition of lethal melanomas remains an unsolved problem in the field. In this study, we begin to address this question. Through molecular profiling, we identified "low" and "high" grade forms of primary melanoma that were (i) recognizable at the earliest stages of melanoma, (ii) reproducible across independent data sets, (iii) significantly associated with known prognostic indicators, and (iv) highly predictive of disease outcome. In recovering these fossilized signatures from formalin-fixed, paraffin-embedded tissue, this study represents the deepest exploration of the expression space within primary melanomas and provides a technical roadmap for overcoming the major stumbling block in melanoma genomicsthe lack of frozen primary tumors for RNA profiling. In short, we believe that these findings will create new avenues of clinical diagnostics and open up previously obscured vistas in melanoma biology. 


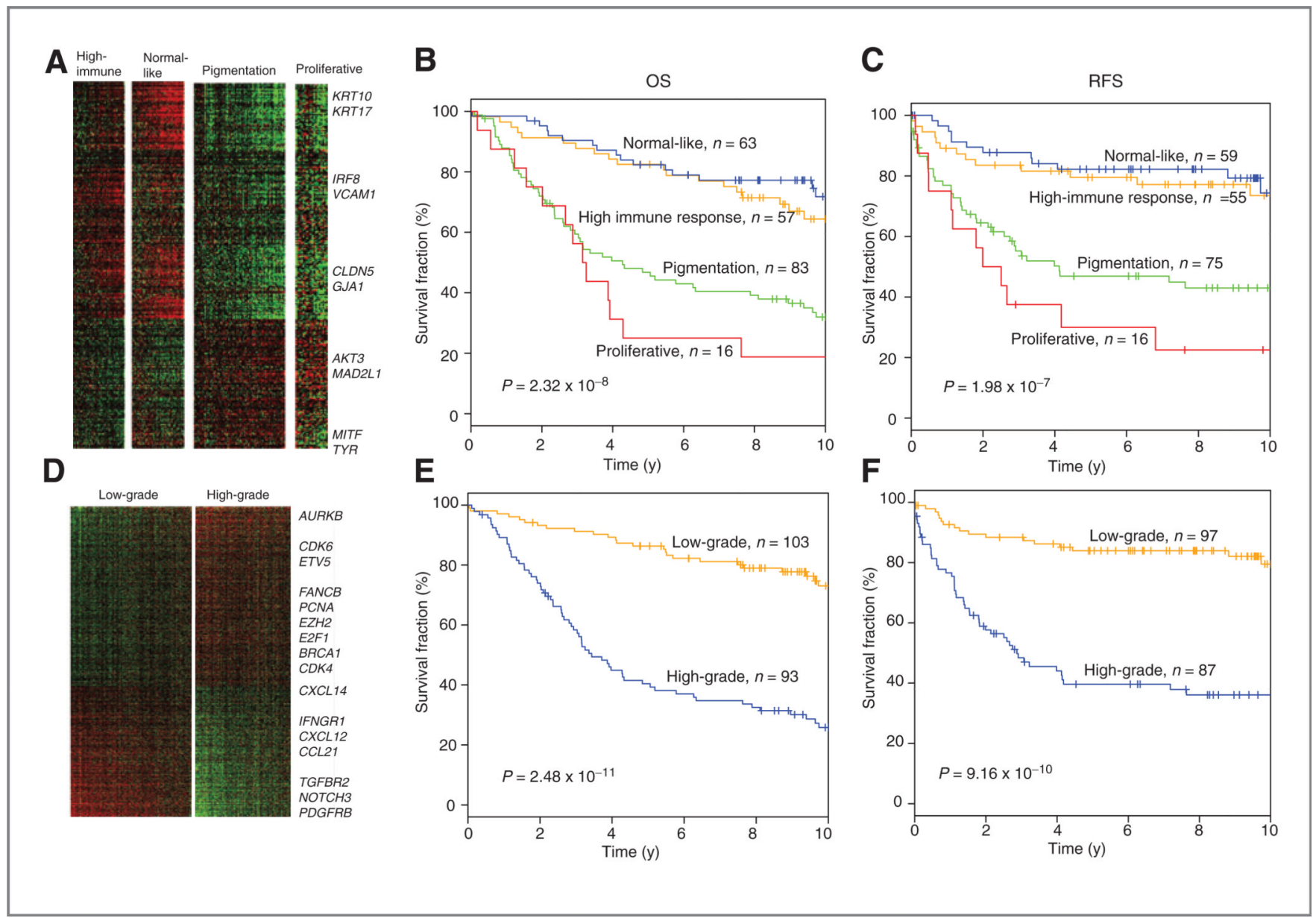

Figure 1.

Gene expression patterns in primary melanomas. A, primary melanomas were classified in the 4 molecular subtypes using a nearest-centroid approach with a heat map displaying upand downregulated genes in their respective class. Kaplan-Meier analyses showing OS (B) and RFS (C) differences between the molecular subtypes. D, heat map of the approximately 1,500 genes that were used to distinguish "high" and "low"-grade primary melanomas. Kaplan-Meier analyses showing significant differences in OS (E) and RFS (F) using molecular grading. 


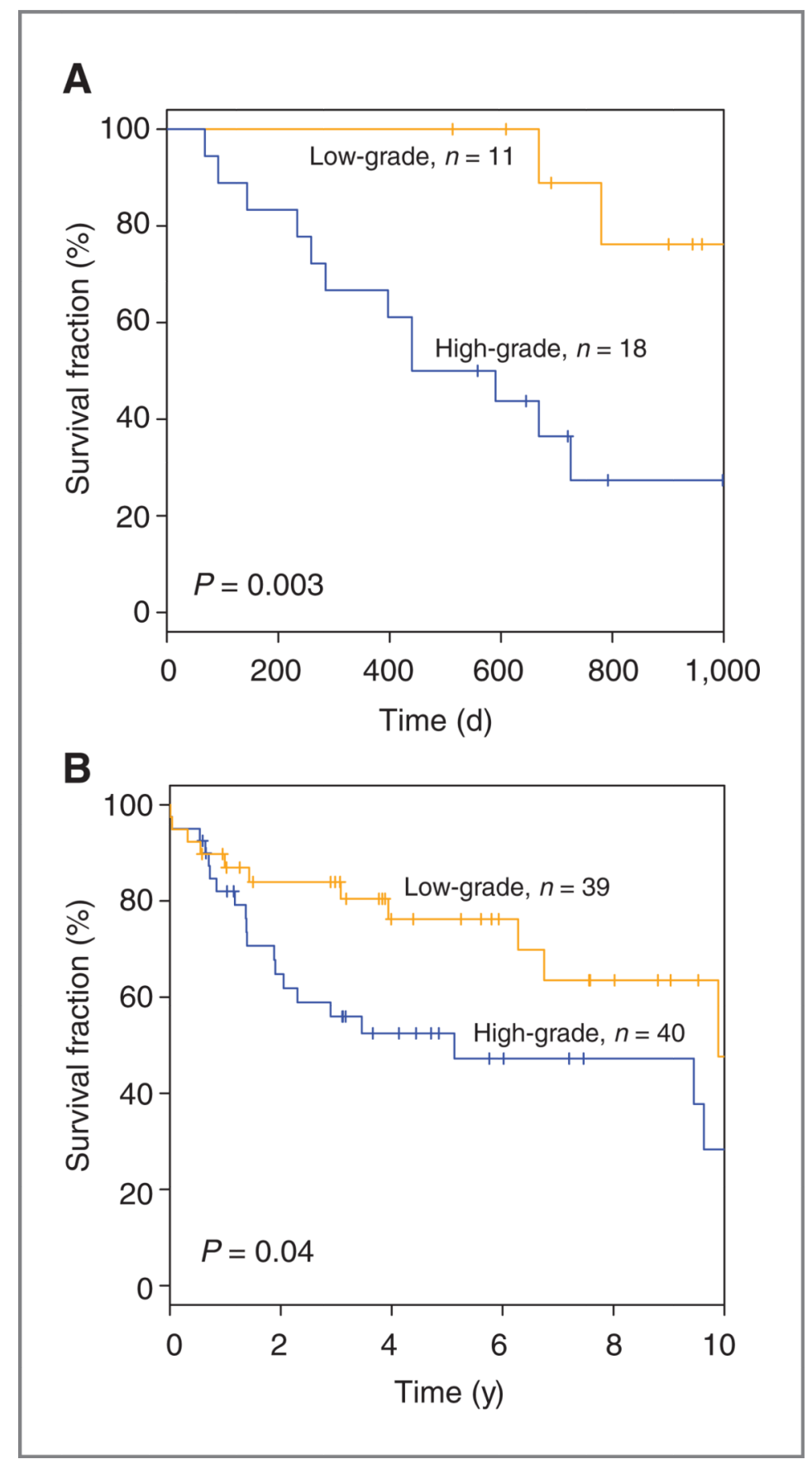

Figure 2.

Molecular grading and Kaplan-Meier analyses for (A) stage III melanomas (Bogunovic and colleagues; ref. 9) and (B) primary melanomas (Winnepenninckx and colleagues; ref. 5) using public data sets. Significant differences in survival rates were found for both cohorts corroborating the findings in the primary melanoma data set. 


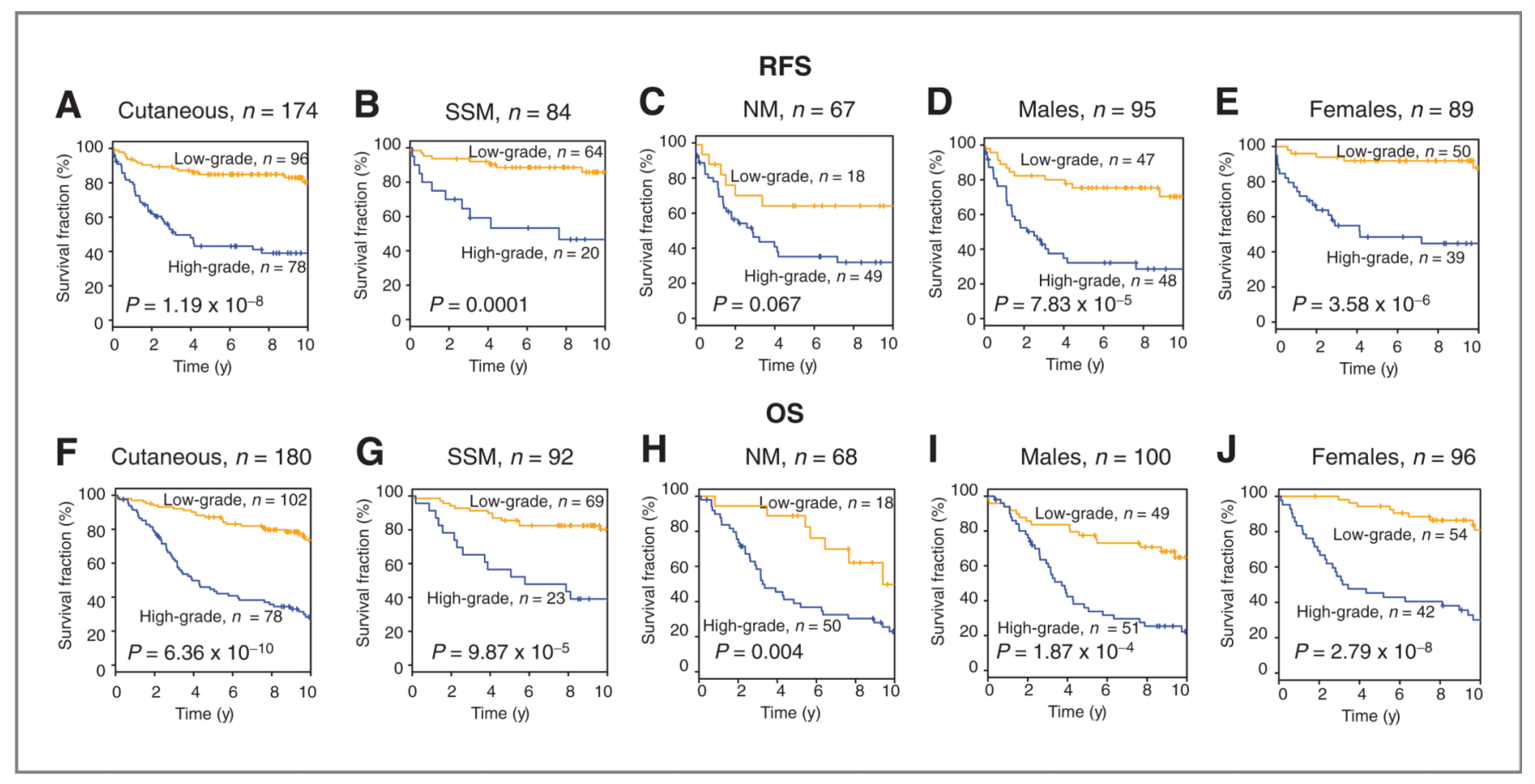

Figure 3.

Survival analysis in subgroups of primary melanoma. Differences in RFS (A-E) and OS(FJ) between high- and low-grade melanomas among various subgroups: A and $\mathrm{F}$, cutaneous melanomas only; B and G, SSM; C and H, NM; D and I, males; and E and J, females. 
A

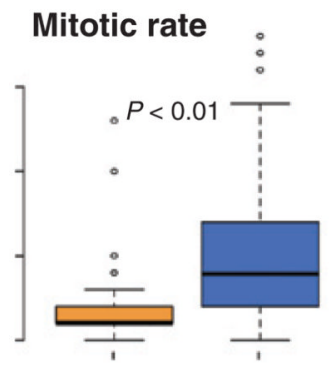

Breslow thickness

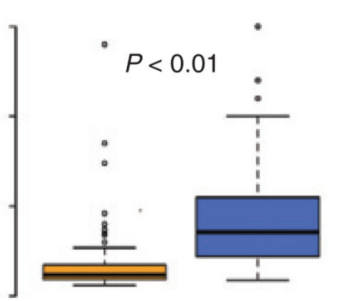

Ulceration

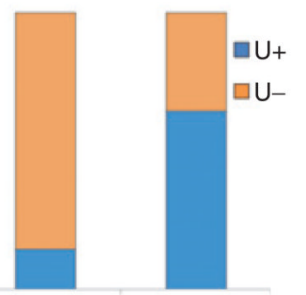

B Melanomas $\geq 2 \mathrm{~mm}(\mathrm{~N}=79)$

Low-grade
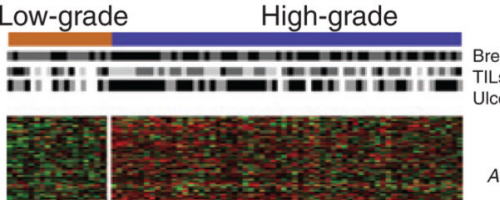

\section{AURKB}

Figure 4.

Features of high-grade melanomas. A, high-grade primary melanomas were significantly associated with increased mitotic rate, thickness, and ulceration. B, heat map showing that both molecular grades were evident in melanomas $\geq 2 \mathrm{~mm}$. C and D, among melanomas greater than $2 \mathrm{~mm}$, molecular grading was still predictive of survival. E, subset KaplanMeier analysis of the Winnepenninckx data set including melanomas $\geq 2 \mathrm{~mm}$ shows significant difference in patient survival. Breslow thickness: black, $>4 \mathrm{~mm}$; gray, 2 to $4 \mathrm{~mm}$; ulcerated, dark gray; and no ulceration, light gray. TILs: brisk, light gray; nonbrisk, dark gray; absent, black; and absent data, white. 

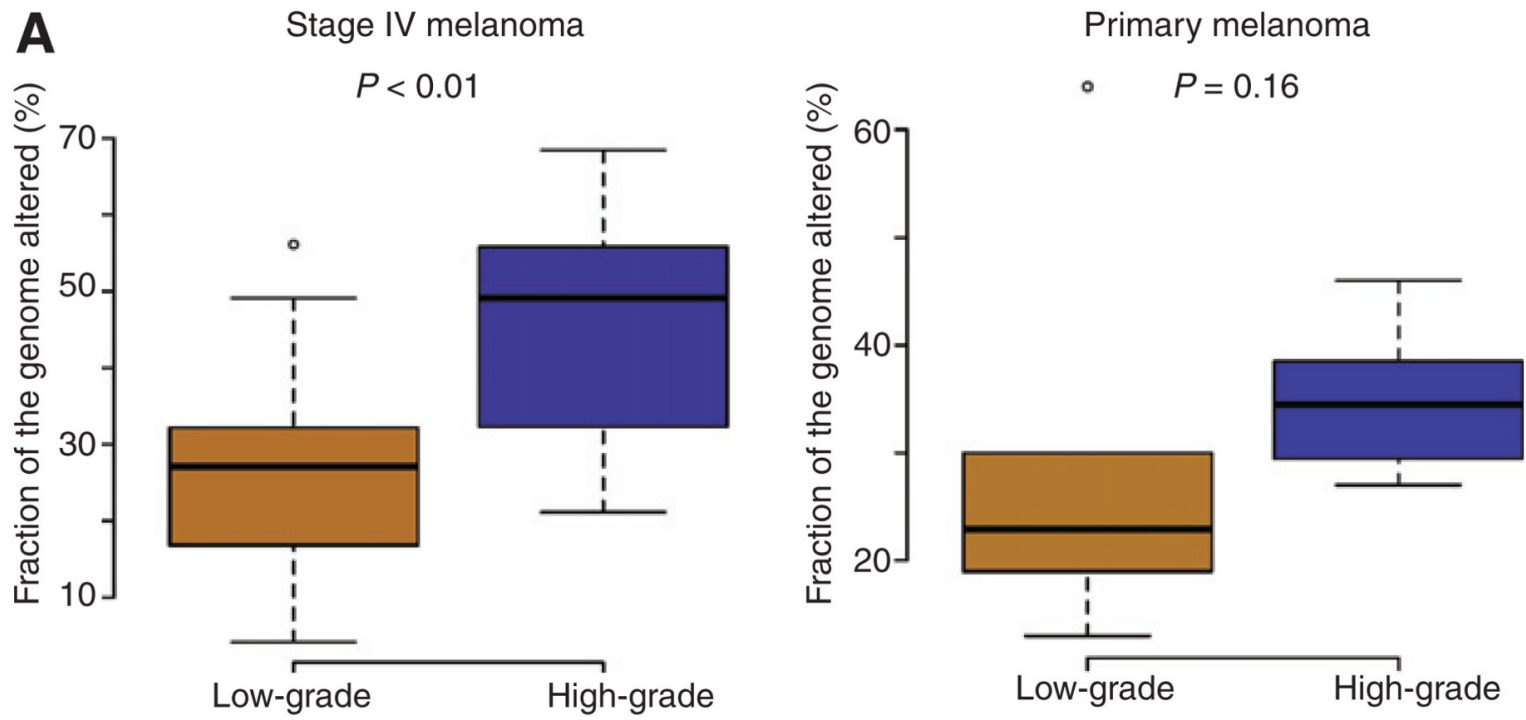

\section{B}

\section{Low}

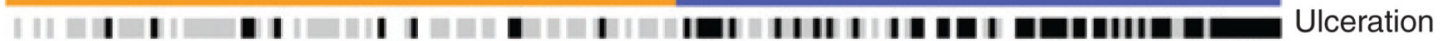

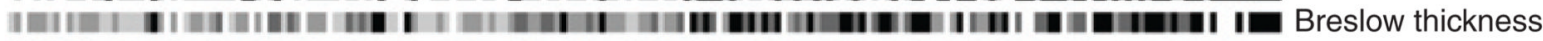

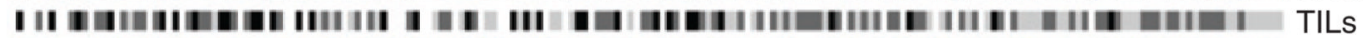

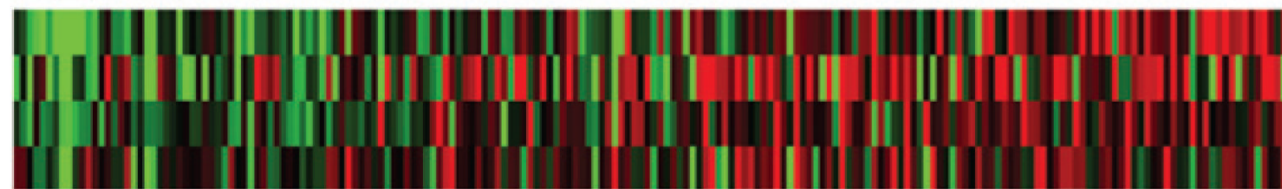
MITF TYR SOX10 MLANA

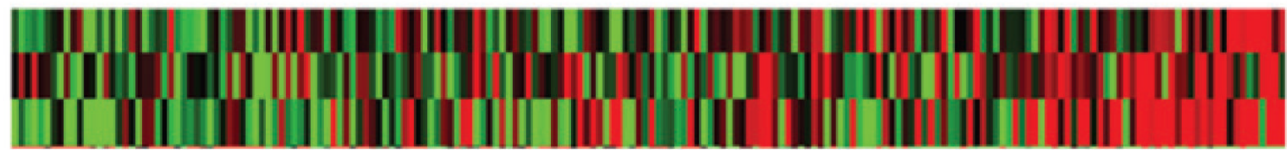

$B R C A 1$

ATM FANCA

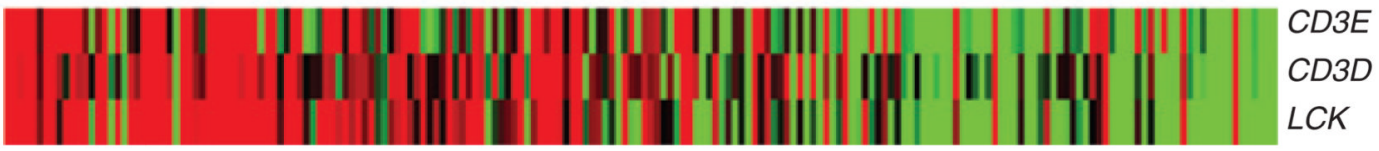

Figure 5.

Biologic correlates of molecular grading. A, high-grade melanomas were associated with more genomic imbalance in both stage IV and primary melanomas. B, ranking of specimens from low- to high-grade molecular profiles revealed parallel increases in MITF-related genes (MITF, TYR, SOX10, and MLANA) and BRCA-related genes (BRCA1, ATM, and $F A N C A$ ). Conversely, there was a diminution of immune-related genes $C D 3 E, C D 3 D$, and $L C K$. 


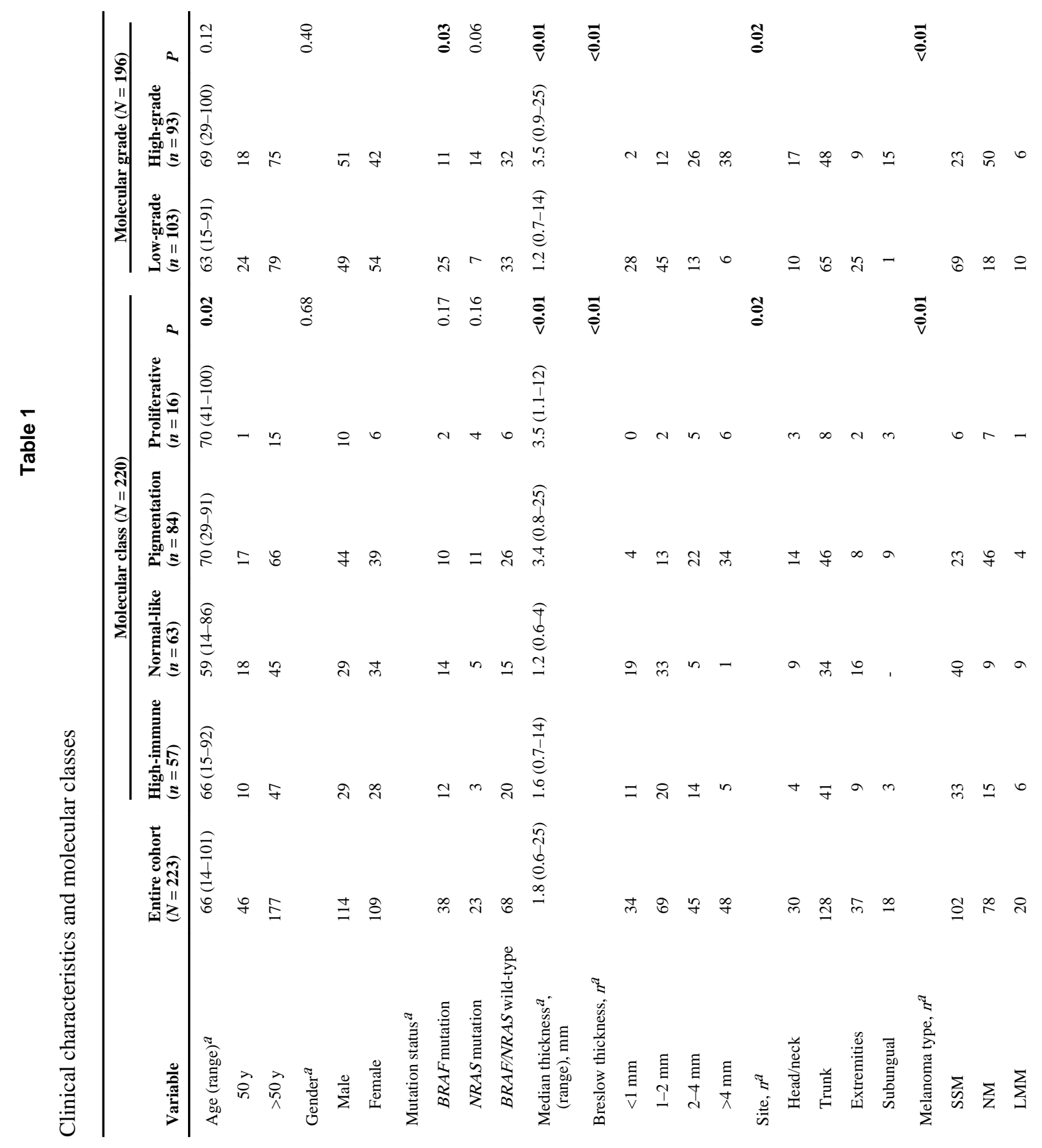




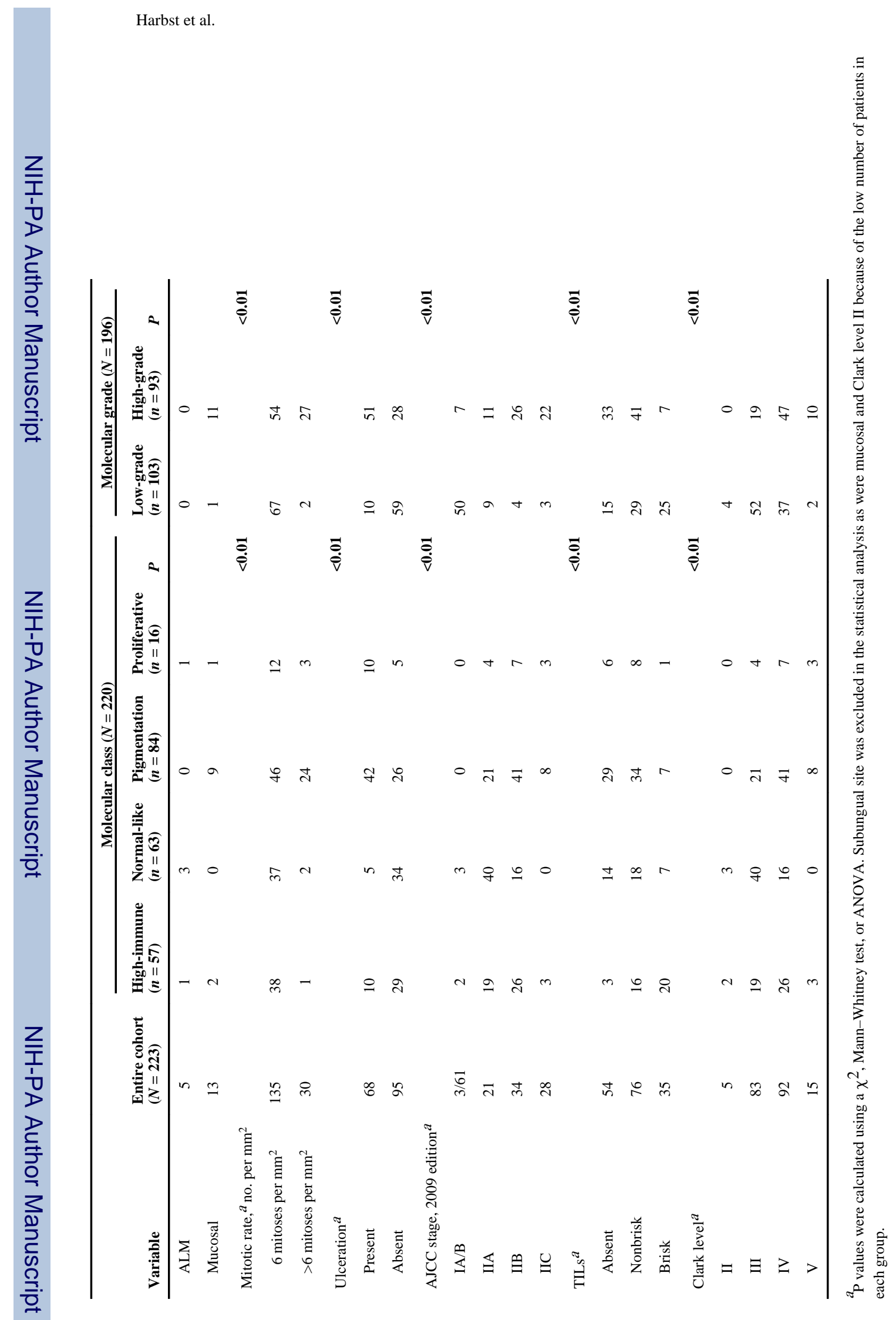

Clin Cancer Res. Author manuscript; available in PMC 2013 August 01. 